\title{
Efficacy of Oil Pulling Therapy
}

\author{
Dr. Seema Diwan ${ }^{1}$, Dr. Abhishek Kandwal ${ }^{2}$, Dr. S L Jethani ${ }^{3}$, \\ Vaibhav Gupta ${ }^{4}$ \\ ${ }^{1}$ MDS, FCFO (Fellowship in Craniofacial Orthodontics) Associate Professor and Head, Department of Dental \\ Sciences, Himalayan Institute of Medical Sciences \\ ${ }^{2}$ MDS (Periodontics) Assistant Professor, Department of Dental Sciences, Himalayan Institute of Medical \\ Sciences; \\ ${ }^{3}$ Dean, Professor and Head of Department of Anatomy. Himalayan Institute of Medical Sciences; \\ ${ }^{4}$ Research Fellow, Cancer Research Institute, Himalayan Institute of Medical Sciences;
}

\begin{abstract}
We report a case report of 34 year old male who came with complaint of halitosis and loose tooth. Examination revealed poor oral hygiene with sub gingival calculus. Diagnosis of chronic generalized gingivitis with localized periodontitis was made. A strict oral hygiene protocol was developed for maintaining oral hygiene status and oil pulling therapy was started as an integral part of the therapy. We found a significant improvement in mobility of tooth due to bone regeneration and improved halitosis scores. From this case report, it is evident that oil pulling therapy as an adjunct to oral hygiene protocolis efficient in treating periodontal diseases in future.
\end{abstract}

Key words: Halitosis, Oil pulling therapy, Periodontitis,

\section{Introduction}

Prevention is always better than cure. Hence, more population is inclining towards preventive measures for the treatment. Oil pulling therapy is one of the preventive methods which involve the use of pure oils as antibacterial agents for inhibiting harmful bacteria, fungus and other organisms of the mouth, teeth, gums and throat [1]. It has been mentioned in the Ayurvedic text CharakaSamhita (Sutrasthana 5, 78-80) where it is called KavalaGandoosha/KavalaGraha[2]. Oil pulling therapy has been used extensively as a traditional Indian folk remedy for many years to prevent decay, oral malodor, bleeding gums, dryness of the throat, and for strengthening teeth, gums, and the jaw[3]. Concept of oil pulling therapy was first familiarized by Dr F. Karach in 1990[4]. Although there has been many studies who have proved effect of oil pulling therapyin reducing plaque, gingivitis and periodontitis $[5,6,7,8$,$] but there is no literature or scientific proof to accept oil pulling as$ an adjunct therapy. The present case report clearly demonstrates soft and hard tissue regeneration by using oil pulling therapy as an adjunct to oral hygiene protocol.

\section{Case Report}

A 34-year-oldmale presentedwith the complaint of a loose lower back tooth and oral malodor in the department of dentistry. Patient gave a positive history of bleeding gums and halitosis since five years. Medical and family histories werenoncontributory. A complete and comprehensive periodontal examination was initiated which revealed a poor oral hygiene with a score of $4.6(3.5+1.16)$ for oral hygiene index (OHI Green and vermillion)Gingival status was recorded by loe and silnessindex which gave a score of 2.2, indicatingsevere gingival inflammation (Table. 1). There was generalized loss of Stippling on attached gingiva and the marginal gingiva wereround,bulbous, erythematous, fragile and bleeding on probing was present. Next periodontal probing was done and CAL (Clinical Attachment Level) scores were recorded (M B D L) scores were recorded at four siteson all teeth.On clinical examination, it was found that patient had deep periodontal pockets in tooth $36,37,46,47$ and angular bone defect.A diagnosis of chronic generalizedgingivitis with localized periodontitis in respect to teeth no. 16, 18,26, 36-38,46-48, was made (Fig. 1).Halitosis was also assessed by two examiners and average was taken out for three visits. Score of 4 was given indicating strong malodor.Patient was not ready for any surgical intervention hence the required periodontal flap surgery was not initiated.Instead a strict oral hygiene protocol was developed for maintaining oral hygiene status and oil pulling therapy was started as an integral part of the same. Patient was explained the procedure of oil pulling therapy thoroughly. He was advised to usea tablespoon of sesame oil to be sipped, sucked and pulled between the teeth for 10-15 minutes empty stomach. Afterwards, the viscous oil was to be spitted out. This was followed by tooth brushing and rinsing of the mouth with plain water. After recording all baseline data and taking OPG record, adeep through root planning and sub gingival scaling was done under local anesthesia and patient was instructed to start oil pulling therapy.Subgingival scaling was done one quadrant per week. After completing the supra and sub gingival scaling, patient was instructed to be on monthly recall for reinforcement of oral hygiene measure and evaluation 
of treatment. Patient was guided for oral hygiene measures every month and a OPG was decided to be taken at $6^{\text {th }}$ month recall. At same visit oral hygiene indices were repeated along with a comprehensive periodontal examination. There was significant improvement in oral hygiene score, gingival score andhalitosis grade. On comparing OPG a clinically significant bone regeneration was observed which was correlated with improvement in mobility and CAL scores(Fig. 2), (Table. 1).

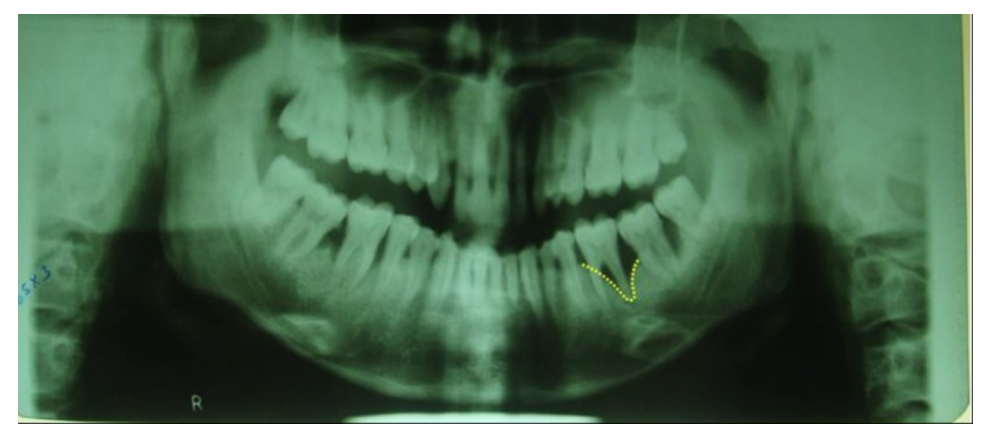

Fig. (1): OPG at baseline showing areas of localized periodontitis.

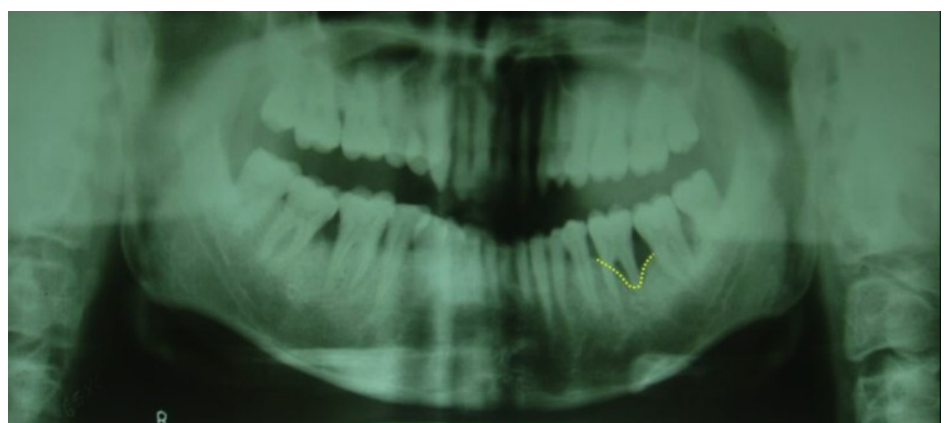

Fig. (2) OPG at $6^{\text {th }}$ month recall depicting bone formation.

\section{Mechanism Of Action}

Sesame oil is a vegetable fat which reacts with salivary alkalis like bicarbonates causes saponification by acting as an emulsifying agent. This soapy layer prevents bacterial adhesion to tooth surface and coaggregation of bacteria layer in biofilm [9].

\section{Discussion}

There are very few clinical trials evaluating the effect of oil pulling therapy on oral health. Amithet al, [8] reported significant improvement in plaque scores, while Sharath A et., al [10] concluded its effectiveness in inhibiting microorganisms. Our case report also showed results consistent with the earlier mentioned authors. There was a significant improvement in plaque, gingival scores. Also, soft and hard tissue regeneration was observed in present case in terms of CAL improvementand radiographic evidence of bone formation. In routine clinics, we always use chlorhexidine as a gold standard for improving health of periodontal tissues. Because of staining and bitter taste of chlorhexidine, patient compliance is not very good always. But oils used in oil pulling therapy are non-staining, easily accessible even available in homes. Hence, making compliance much better.

Therefore, we may conclude that oil pulling therapy along with oral hygiene protocol is effective in controlling periodontal diseases in both types of patients who are notwilling for periodontal surgery ormedically compromised patients.

\begin{tabular}{|c|c|c|c|c|c|c|}
\hline Tooth no & \multicolumn{3}{|c|}{ Baseline } & \multicolumn{2}{c|}{$\mathbf{6}^{\text {th }}$ month } \\
\hline & $\begin{array}{l}\text { CAL } \\
\text { (M,B,D,L) }\end{array}$ & Mobility & $\begin{array}{l}\text { Furcation } \\
\text { grade }\end{array}$ & $\begin{array}{l}\text { CAL } \\
(\mathrm{M}, \mathrm{B}, \mathrm{D}, \mathrm{L})\end{array}$ & $\begin{array}{l}\text { Mobility } \\
\text { grade }\end{array}$ \\
\hline 16 & $6,6,5,5$ & 1 & & $5,4,5,5$ & 0 & \\
\hline 18 & $5,7,8,6$ & 1 & & $5,5,6,5$ & 0 & \\
\hline 26 & $6,7,5,5$ & 1 & & $5,6,5,5$ & 1 & $2 \mathrm{~b}$ \\
\hline 36 & $6,6,8,7$ & 3 & 3 & $5,5,6,5$ & 1 & $2 \mathrm{a}$ \\
\hline 37 & $6,5,8,8$ & 2 & $2 \mathrm{a}$ & $5,5,6,6$ & 1 & $2 \mathrm{a}$ \\
\hline 38 & $5,4,3,3$ & 1 & & $4,4,3,3$ & 1 & $2 \mathrm{a}$ \\
\hline 46 & $6,9,9,8$ & 2 & $2 \mathrm{~b}$ & $5,6,6,5$ & 1 & 1 \\
\hline 47 & $9,6,5,4$ & 2 & $2 \mathrm{a}$ & $6,6,5,5$ & & 1 \\
\hline 48 & $6,5,4,4$ & 1 & & $5,5,4,4$ & 0 & \\
\hline
\end{tabular}

Table (1):Baseline and $6^{\text {th }}$ month data of CAL, Mobility and Furcation grades. 


\section{References}

[1]. The health benefits of oil pulling. Available from: http://www.in5d.com/oil-pulling.html[Last accessed on 2013 Feb 28].

[2]. Available from http://en.wikipedia.org/wiki/oil-pulling. [accessedon 2013,july 20].

[3]. Is oil pulling really good for you? Available from: http://www thebeautybrains.com/2012/10/20/is-oil-pulling-really-goodforyou.[Last accessed on 2012 Oct 20].

[4]. Avalablefrom http://www.oilpulling.com [accessed on 2013,july 20].

[5]. Robertson PB, Armitage GA, Buchanan SA, Targgat EV. The design of trials to test the efficacy of plaque control agents for periodontal diseases in humans. J Dent Res 1989;68:1667-71.

[6]. Stoltze K, Bay L. Comparison of a manual and a new electric toothbrush for controlling plaque and gingivitis. J ClinPeriodontol 1994;21:86-90.

[7]. Putt MS, Kleber CJ, Smith CE. Evaluation of an alum-containing mouthrinse in children for plaque and gingivitis inhibition during 4 weeks of supervised use. Pediatr Dent 1996;18:139-44.

[8]. Amith HV, Ankola AV, Nagesh L. Effect of oil pulling on plaque and gingivitis. J Oral Health Community Dent 2007;1:12-8.

[9]. AsokanS, Rathan J, MuthuMS,Rathna PV, Emmadi P, Raghuraman C et al. Effect of oil pulling on Streptococcus mutans count in plaque and saliva using Dentocult SM strip mutanstests:A randomized controlled triple blind study. J Indian SOC PedodPrev Dent 2008;26;12-7.

[10]. Asokan S, Rathinasamy TK, Inbamani N, Menon T, Kumar SS, Emmadi P, et al. Mechanism of oil-

[11]. pulling therapy-In vitro study. Indian J Dent Res 2011;22:34-7. 